\title{
Predictors of Pathologic Response After Total Neoadjuvant Therapy in Patients With Rectal Adenocarcinoma: A National Cancer Database Analysis
}

David M. McDermott ${ }^{1}$, Sarah A. Singh ${ }^{1}$, Paul B. Renz ${ }^{2}$, Shaakir Hasan ${ }^{3}$, Josh Weir ${ }^{1}$

1. Radiation Oncology, West Virginia University School of Medicine, Morgantown, USA 2. Radiation Oncology, Allegheny Health Network, Pittsburgh, USA 3. Radiation Oncology, New York Proton Center, New York, USA

Corresponding author: Sarah A. Singh, sarah.amrita.singh@gmail.com

\section{Abstract \\ Purpose/objectives}

Induction chemotherapy followed by chemoradiation and surgical resection in rectal cancer, known as total neoadjuvant therapy (TNT), is associated with improved pathologic complete response (pCR) rates. The National Cancer Database was utilized to identify factors associated with pCR and survival following treatment with TNT compared to standard neoadjuvant chemoradiation (nCRT).

\section{Materials/methods}

The National Cancer Database was queried from 2004 to 2015 for patients with locally advanced, nonmetastatic rectal cancer. We identified 16,299 patients receiving neoadjuvant chemotherapy and radiation followed by definitive surgical resection. Patients were stratified by treatment received, either TNT $(n=350)$ or nCRT ( $\mathrm{n}=15,949)$. Multivariate binomial regression analysis and propensity matching were used to evaluate predictors of pCR. Kaplan-Meier and Cox multivariate analysis of survival were performed.

\section{Results}

Median follow-up was 38 months vs 53 months in the TNT vs nCRT groups, respectively. There were more patients with T4 or node-positive disease in the TNT group. There was a trend towards improved pCR in the TNT group ( $\mathrm{p}=0.053$ ). Patients achieving pCR had improved 5 -year overall survival (OS) of $85.1 \%$. The 5 -year OS was not improved for TNT $(76.2 \%)$ over nCRT $(69.9 \%)(\mathrm{p}=0.19)$. Pelvic nodal $\mathrm{pCR}$ was significantly higher in the TNT group (72\%). When stratified by clinical stage, patients with cT3 $(\mathrm{p}=0.038)$ or $\mathrm{cN} 1(\mathrm{p}=0.049)$ disease had improved OS with TNT.

\section{Conclusions}

Review began 07/12/2021 Review ended 08/08/2021 Published 08/16/2021

\section{() Copyright 2021}

McDermott et al. This is an open access article distributed under the terms of the Creative Commons Attribution License CC-BY 4.0., which permits unrestricted use, distribution, and reproduction in any medium, provided the original author and source are credited.
Compared to nCRT, TNT is correlated with higher rates of complete pelvic nodal clearance in patients with locally advanced rectal adenocarcinoma. The use of TNT showed improved survival in patients with cT3 and cN1 disease, indicating a potential benefit for patients with less advanced disease.

Categories: Radiation Oncology, Oncology

Keywords: total neoadjuvant therapy, colo rectal cancer, national cancer database and seer analyses, non metastatic colo-rectal, chemoradiation therapy

\section{Introduction}

Nearly 50,000 people are diagnosed with rectal cancer every year in the United States and colorectal cancer is the third leading cause of cancer death nationwide [1]. The treatment paradigm for stage II-III locally advanced rectal cancer involves neoadjuvant chemoradiation (nCRT) followed by surgery and adjuvant chemotherapy [2]. The German rectal study [3], which established pre-operative chemoradiation as a standard of care for stage II-III rectal adenocarcinoma, showed high rates of distant recurrence at 10 years (30\%) compared to local recurrence (7\%). It has been hypothesized that higher rates of distant recurrence may be the result of delays in treatment with systemic therapy and poor compliance in the adjuvant setting. Previous studies have demonstrated that more than half of patients receive no or incomplete courses of chemotherapy following surgery, primarily due to disease progression, patient refusal, and postoperative complications which occur in approximately $20 \%$ of patients $[4,5]$.

Numerous studies have shown that pathologic tumor response rates following nCRT for rectal cancer are an important prognostic factor for local and distant disease-free survival [6,7]. This is particularly true for rates of pathologic nodal response (ypN) where posttreatment pathology stage (yp) N0, N1, and N2 are associated with 10 -year disease-free survival rates of $84 \%, 59 \%$, and $28 \%$, respectively [8]. 
Total neoadjuvant therapy (TNT), consisting of induction chemotherapy followed by concurrent chemoradiation and surgical resection, is a therapeutic strategy proposed to better target micrometastatic disease through early exposure to high dose chemotherapy and increased compliance with systemic treatment [9]. Induction chemotherapy in this setting is typically an oxaliplatin-based, multi-agent regimen administered for six to eight cycles prior to concurrent chemoradiation as seen in currently ongoing clinical trials NRG GI-002 (clinicaltrials.gov, NCT 02921256) and PROSPECT (clinicaltrials.gov, NCT 01515787). The use of TNT has been shown to improve pathologic complete response (pCR) rates, however, there is a lack of data examining whether or not this directly translates into improved disease outcomes $[9,10]$. We utilized the National Cancer Database (NCDB) to evaluate the effect of TNT on pathologic treatment response and to determine whether or not this translates into a survival benefit. We also sought to determine the clinical factors associated with the utilization of TNT.

\section{Materials And Methods Patient selection}

We utilized the data set of the National Cancer Database (NCDB) to identify our study population consisting of patients diagnosed with rectal cancer from 2004-2015. The institutional review board deemed this study exempt due to the use of de-identified, population-based patient data. A consolidated standard of reporting trials (CONSORT) diagram is provided to show the selection criteria (Figure 1). Patients were excluded if they had stage I disease (based on the provided American Joint Committee on Cancer [AJCC] staging), metastatic disease, or incomplete clinical and pathologic staging information. We further excluded patients that did not undergo radiation treatment or definitive surgery. We then excluded patients with non-adenocarcinoma histology, if it was not known whether they received single or multi-agent chemotherapy, if surgery was performed prior to radiation or chemotherapy initiation, or if the length of time from diagnosis to surgery was unknown as we would be unable to determine the sequencing of treatment. We also excluded patients who were treated with non-standard radiation doses ( $<20 \mathrm{~Gy}$ or $>60 \mathrm{~Gy})$ or fractionation regimens $(<5$ or $>40$ fractions), had prolonged delay from diagnosis to treatment initiation ( $>120$ days), were treated with a radiation modality other than protons or photons, or had limited post-surgical follow-up within one month of their surgery date to account for immortal time bias. This resulted in 27,112 patients prior to treatment stratification.

The remaining patients were then stratified into two treatment groups: the TNT group and the nCRT group. Patients starting radiation therapy $>90$ days after initiating multi-agent chemotherapy were included in the TNT group, whereas those starting radiation treatment within 30 days of single-agent chemotherapy were included in the nCRT group. After applying all exclusion criteria, 350 patients in the TNT group and 15,949 patients in the nCRT group were included in the final analysis. Pathologic response was determined using clinical and pathological staging information.

\section{Statistics}

The primary outcome was overall pCR rate, as well as the pCR rate of the primary tumor (ypT0) and nodes (ypN0). We also evaluated overall survival (OS) which was calculated from the date of diagnosis to the date of last contact or death, as we are unable to directly assess the distant metastasis rate using the NCDB dataset. Additional factors examined include patient age, gender, insurance coverage, residence income data, urban or rural residence, academic versus non-academic treatment facility, Charlson/Deyo comorbidity index, year of diagnosis, grade, clinical and pathologic $\mathrm{T}$ and $\mathrm{N}$ stage based on the AJCC eighth edition, and neoadjuvant rectal cancer (NAR) score. The NAR score is a composite score that predicts overall survival after neoadjuvant treatment for rectal cancer and is based on the pathologic response to therapy [11,12]. A NAR score $>16$ falls into the high-risk category and is associated with worse overall survival compared to scores $<16$. We also evaluated the time from radiation treatment completion to surgery, the total radiation dose administered, length of radiation treatment in days, and time from diagnosis to initiation of chemotherapy. We dichotomized groups based on the median value.

Baseline patient and treatment characteristics were compared between TNT and nCRT groups using $\chi 2$ test. Bivariate logistic regression models were used to evaluate the association between independent variables of interest. Variables that were significant on univariate analysis were included in a multivariate binomial regression analysis. These models were also used to evaluate predictors of pCR within each treatment group. The Kaplan-Meier method was used to assess survival outcomes, and the log-rank test was used to assess statistical significance between groups. The factors that were statistically significant on univariate analysis were entered using stepwise selection for multivariate analysis using Cox proportional hazard models to calculate adjusted hazard ratios for survival.

Propensity score analysis was conducted to account for the lack of randomization between the treatment groups. Variables found to be significantly associated with TNT use on univariate logistic regression were included. Overall survival was then determined using the Cox proportional hazards model adjusting for propensity score. The matched groups were balanced based on a standardized difference of $<0.1$ between factors. Any p-value less than 0.05 was considered statistically significant. The hazard ratios (HRs), odds ratios (ORs), and 95\% confidence intervals (CIs) are reported. Statistical analysis was performed using IBM SPSS version 24 (IBM cooperation, Armonk, NY). 


\section{Cureus}

\section{Results}

We identified a total of 16,299 patients diagnosed with locally advanced rectal adenocarcinoma meeting our inclusion criteria, with 15,949 in the nCRT group, and 350 in the TNT group (Figure 1). Baseline patient characteristics are outlined in Table 1. The median patient age was 54 years (Interquartile range (IQR): 4663) in the TNT group and 62 years (IQR: 53-71) in the nCRT group. The distribution of gender, insurance coverage, and tumor grade were similar between the groups. Patients treated in the TNT group were more likely to be younger $(\mathrm{p}<0.001)$, treated at an academic facility $(\mathrm{p}<0.001)$, have a higher income $(\mathrm{p}<0.001)$, have a lower comorbidity score $(\mathrm{p}<0.001)$, and more recent treatment $(\mathrm{p}<0.001)$. There were proportionally more patients with cT4 disease ( $17 \%$ vs $8 \%$ ), node-positive disease ( $81 \%$ vs $50 \%$ ), and cN2 disease ( $20 \%$ vs $7 \%$ ), in the TNT group versus the nCRT group, respectively.

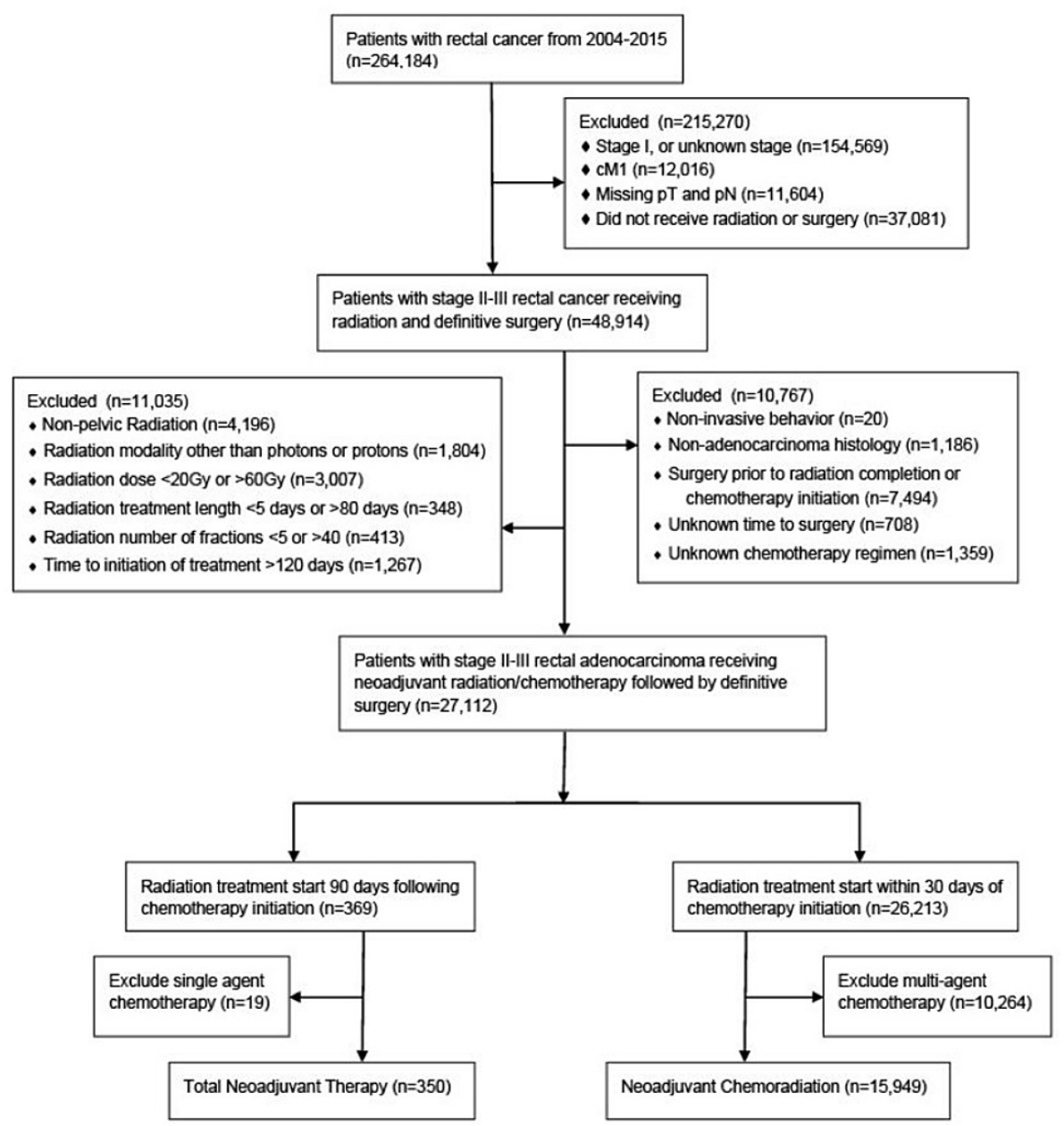

FIGURE 1: Consolidated Standards of Reporting Trials (CONSORT) diagram showing patient selection

\begin{tabular}{|c|c|c|}
\hline Characteristic & nCRT (N=15,949) n (\%) & TNT $(\mathrm{N}=35$ \\
\hline \multicolumn{3}{|l|}{ Age (years) } \\
\hline$\leq 65$ & $9594(60 \%)$ & $288(82 \%)$ \\
\hline$>65$ & $6355(40 \%)$ & $62(18 \%)$ \\
\hline \multicolumn{3}{|l|}{ Gender } \\
\hline Male & 9914 (62\%) & $213(61 \%)$ \\
\hline Female & 6035 (38\%) & 137 (39\% \\
\hline
\end{tabular}




\section{Cureus}

\begin{tabular}{|c|c|c|c|c|}
\hline Community & $8083(51 \%)$ & 55 (16\%) & & \\
\hline Academic & 7301 (46\%) & $256(73 \%)$ & & \\
\hline Unknown & $565(3 \%)$ & $39(11 \%)$ & & \\
\hline Insurance & & & & 0.15 \\
\hline Uninsured & 744 (5\%) & $9(3 \%)$ & & \\
\hline Insured & 15017 (94\%) & $338(96 \%)$ & $3(1 \%)$ & \\
\hline Unknown & 188 (1\%) & & & \\
\hline Income (\$) & & $79(23 \%)$ & & $<0.001$ \\
\hline$<48,000$ & $6763(42 \%)$ & $271(77 \%)$ & & \\
\hline$\geq 48,000$ & 9055 (57\%) & $0(0 \%)$ & & \\
\hline Unknown & $131(1 \%)$ & & & \\
\hline Population & & $308(88 \%)$ & & \\
\hline Urban & $15167(95 \%)$ & $5(1 \%)$ & & \\
\hline Rural & 419 (3\%) & $37(11 \%)$ & & $<0.001$ \\
\hline Unknown & $363(2 \%)$ & & & \\
\hline Comorbidity Score & & $301(86 \%)$ & & \\
\hline 0 & $12358(78 \%)$ & $49(14 \%)$ & & \\
\hline$\geq 1$ & $3591(22 \%)$ & & & $<0.001$ \\
\hline Year & & $99(28 \%)$ & & \\
\hline 2004-2012 & $9461(59 \%)$ & $251(72 \%)$ & & \\
\hline 2013-2015 & $6488(41 \%)$ & & & $<0.001$ \\
\hline Grade & & 277 (79\%) & & \\
\hline $1-2$ & $11890(75 \%)$ & $36(10 \%)$ & & \\
\hline 3 & $1688(10 \%)$ & 37 (11\%) & & 0.072 \\
\hline Unknown & $2371(15 \%)$ & & & \\
\hline Clinical T stage & & $18(5 \%)$ & & \\
\hline cT1-T2 & 800 (5\%) & 264 (76\%) & & \\
\hline cT3 & $13684(86 \%)$ & $60(17 \%)$ & & $<0.001$ \\
\hline cT4 & $1249(8 \%)$ & $8(2 \%)$ & & \\
\hline Unknown & $216(1 \%)$ & & & \\
\hline Clinical N stage & & 64 (18\%) & & \\
\hline cNO & 7602 (48\%) & $215(61 \%)$ & & \\
\hline $\mathrm{cN} 1$ & $6851(43 \%)$ & $69(20 \%)$ & & $<0.001$ \\
\hline cN2 & $1141(7 \%)$ & $2(1 \%)$ & & \\
\hline Unknown & $355(2 \%)$ & & & \\
\hline
\end{tabular}

\section{TABLE 1: Baseline patient characteristics}

nCRT: neoadjuvant chemoradiation; TNT: total neoadjuvant therapy 
The treatment characteristics for patients are outlined in Table 2. The median time from radiation treatment completion to surgery was 61 days for the TNT group and 56 days for the nCRT group (OR=1.49, $\mathrm{p}<0.001)$, which was not significant on multivariate analysis ( $\mathrm{p}=0.6$ ). The median total radiation dose (50.4 Gy) and time to initiation of chemotherapy (33 days) were similar between groups. A majority of patients had a total radiation dose of between $50 \mathrm{~Gy}$ to $54 \mathrm{~Gy}$ with $72.6 \%$ vs. $77 \%$ in the TNT and nCRT groups, respectively. Short course radiation to $25 \mathrm{~Gy}$ was administered for $2.6 \%$ of patients in the TNT group and $0.2 \%$ of patients in the nCRT group. Radiation doses $>50.4$ were used to treat $8 \%$ vs. $13 \%$ of patients in the TNT and nCRT groups, respectively $(\mathrm{OR}=0.58, \mathrm{p}=0.005)$, although this was not significant on multivariate analysis $(\mathrm{OR}=0.69$, $\mathrm{p}=0.08)$.

\begin{tabular}{|c|c|c|c|c|c|c|c|}
\hline \multirow{2}{*}{$\begin{array}{l}\text { Treatment Characteristics } \\
\text { Characteristic }\end{array}$} & \multirow[b]{2}{*}{$\begin{array}{l}\text { nCRT } \\
(\mathrm{N}=15,949) \\
\mathrm{n}(\%)\end{array}$} & \multirow[b]{2}{*}{$\begin{array}{l}\text { TNT } \\
(\%)\end{array}$} & \multirow[b]{2}{*}{$\mathrm{n}$} & \multirow[b]{2}{*}{ OR $[95 \% \mathrm{Cl}]$} & \multirow[b]{2}{*}{$\begin{array}{l}P \text { - } \\
\text { value }\end{array}$} & \multicolumn{2}{|c|}{ Propensity Match } \\
\hline & & & & & & OR $[95 \% \mathrm{Cl}]$ & value \\
\hline \multicolumn{8}{|l|}{ Pathologic T Stage } \\
\hline урТ0 & $2372(15 \%)$ & $64(18 \%)$ & & reference & & & \\
\hline урі1 & $1200(8 \%)$ & $23(1 \%)$ & & $\begin{array}{l}0.71[0.44- \\
1.15]\end{array}$ & 0.10 & & \\
\hline ур12 & $4215(26 \%)$ & $98(28 \%)$ & & $\begin{array}{l}0.86 \text { [0.63- } \\
1.19]\end{array}$ & 0.36 & & \\
\hline урТ3 & $6991(44 \%)$ & $141(40 \%)$ & & $\begin{array}{l}0.75[0.55- \\
1.01]\end{array}$ & 0.06 & & \\
\hline урТ4 & $677(4 \%)$ & $19(5 \%)$ & & $\begin{array}{l}1.04[0.62- \\
1.75]\end{array}$ & 0.88 & & \\
\hline Unknown & $494(3 \%)$ & $5(2 \%)$ & & $\begin{array}{l}0.38[0.15- \\
0.94]\end{array}$ & 0.04 & & \\
\hline \multicolumn{8}{|l|}{ Pathologic T Response } \\
\hline урТ+ & $12889(85 \%)$ & $275(82 \%)$ & & reference & & reference & \\
\hline урТ0 & $2357(15 \%)$ & $62(18 \%)$ & & $\begin{array}{l}1.23[0.93- \\
1.63]\end{array}$ & 0.14 & $\begin{array}{l}1.26[0.94- \\
1.68]\end{array}$ & 0.13 \\
\hline \multicolumn{8}{|l|}{ Pathologic N Stage } \\
\hline ypNO & $11272(71 \%)$ & $258(73 \%)$ & & reference & & & \\
\hline ypN1 & $3171(20 \%)$ & $59(17 \%)$ & & $\begin{array}{l}0.81[0.61- \\
1.08]\end{array}$ & 0.16 & & \\
\hline ypN2 & $1183(7 \%)$ & $31(9 \%)$ & & $\begin{array}{l}1.15[0.79- \\
1.67]\end{array}$ & 0.48 & & \\
\hline Unknown & $323(2 \%)$ & $2(1 \%)$ & & $\begin{array}{l}0.27 \text { [0.07- } \\
1.09]\end{array}$ & 0.07 & & \\
\hline \multicolumn{8}{|l|}{ Pathologic N Response } \\
\hline ypN+ & $2848(36 \%)$ & 79 (28\%) & & reference & & reference & \\
\hline ypNO & $5024(64 \%)$ & $204(/ 2 \%)$ & & $\begin{array}{l}1.46[1.12- \\
1.91]\end{array}$ & 0.005 & $\begin{array}{l}1.53[1.16- \\
2.00]\end{array}$ & 0.003 \\
\hline \multicolumn{8}{|l|}{ pCR } \\
\hline урT+ or ypN+ & $12981(85.8 \%)$ & $283(82.5 \%)$ & & reference & & reference & \\
\hline урTONO & $2151(14.2 \%)$ & $60(17.5 \%)$ & & $\begin{array}{l}1.28[0.97- \\
1.70]\end{array}$ & $0.08 /$ & $\begin{array}{l}1.34[1.00- \\
1.80]\end{array}$ & 0.053 \\
\hline \multicolumn{8}{|l|}{ Pathologic Response } \\
\hline урT+N+ & 2601 (35\%) & $71(26 \%)$ & & reference & & & \\
\hline
\end{tabular}




\section{Cureus}

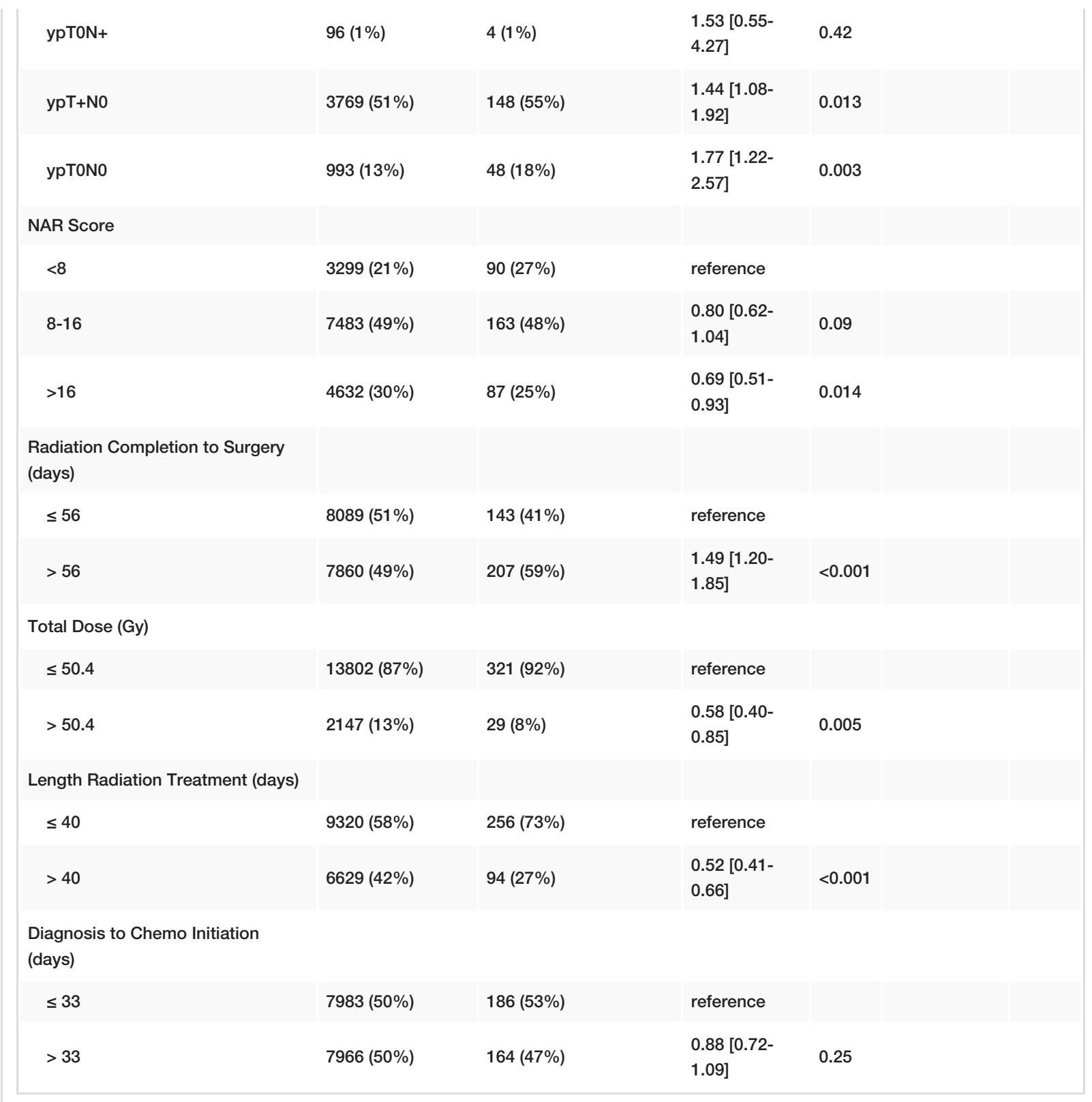

\section{TABLE 2: Treatment characteristics}

nCRT: neoadjuvant chemoradiation; TNT: total neoadjuvant therapy; OR: odds ratio; Cl: confidence interval; pCR: pathologic complete response; NAR: neoadjuvant rectal cancer

There was a trend towards a better $\mathrm{pCR}$ rate in the TNT group ( $17.5 \%$ vs $14.2 \%, \mathrm{p}=0.053$ on propensitymatched analysis [PMA]). The rate of complete pathologic response of the primary tumor (ypT0) was similar in the TNT and nCRT groups ( $18 \%$ vs $15 \%$, respectively, $\mathrm{p}=0.13$ ), however, the rate of nodal pathologic complete response (ypN0) was significantly improved for those receiving TNT on both univariate and PMA ( $72 \%$ vs $64 \%, p=0.003$ ) (Table 2). The rate of nodal $p C R$ for all patients was $65.3 \%$ in those with N1 disease, and $57.3 \%$ for patients with $\mathrm{N} 2$ disease. When stratified by treatment group, the nCRT group had lower rates of nodal $\mathrm{pCR}$ for those with $\mathrm{N} 2$ disease versus $\mathrm{N} 1$ disease $(\mathrm{OR}=0.7, \mathrm{p}<0.001)$, whereas nodal $\mathrm{pCR}$ rates did not differ between $\mathrm{N} 1$ and $\mathrm{N} 2$ patients in the TNT group $(\mathrm{OR}=0.78, \mathrm{p}=0.40)$. Patients in the TNT group were less likely to have a high risk NAR score (>16) compared to the nCRT group ( $25 \%$ vs. $30 \%$, respectively, $\mathrm{p}=0.014$ ).

For the overall study population, improved overall pCR rates were associated with treatment at an academic facility, higher income, being insured, and more recent treatment. The total dose and length of radiation treatment had no effect on $\mathrm{pCR}$, however, time from radiation completion to surgery of $>56$ days was associated with improved $\mathrm{pCR}(\mathrm{OR}=1.21, \mathrm{p}<0.001)$. High tumor grade $(12.8 \%$ for grade $1-2$ and $9.2 \%$ for grade 3 , $\mathrm{p}<0.001$ ), higher $\mathrm{T}$ stage (14.7\% for stage $\mathrm{T} 3$ and $7.1 \%$ for $\mathrm{T} 4$ ), and increasing nodal burden (13.5\% for N1 and $11.8 \%$ for $\mathrm{N} 2$ ) were associated with worse $\mathrm{pCR}$. 


\section{Cureus}

Factors associated with pCR stratified by treatment group are shown in Table 3. For patients receiving nCRT, pretreatment stage T3/T4 disease, increasing nodal burden ( $11, \mathrm{OR}=0.83$; N2, OR=0.69), and longer time from diagnosis to treatment initiation and radiation completion to surgery were associated with worse overall pCR rates. For patients receiving TNT, only cT4 disease was associated with worse overall pCR rates $(\mathrm{OR}=0.2, \mathrm{p}=0.02)$.

\begin{tabular}{|c|c|c|c|c|}
\hline \multirow{2}{*}{ Characteristic } & \multicolumn{2}{|l|}{ nCRT group } & \multicolumn{2}{|l|}{ TNT group } \\
\hline & OR [95\% Cl] & P-value & OR [95\% Cl] & P-value \\
\hline \multicolumn{5}{|l|}{ Age (years) } \\
\hline$\leq 65$ & reference & & reference & \\
\hline$>65$ & $1.06[0.96-1.16]$ & 0.24 & $1.43[0.71-2.84]$ & 0.32 \\
\hline \multicolumn{5}{|l|}{ Gender } \\
\hline Male & reference & & reference & \\
\hline Female & $1.07[0.98-1.18]$ & 0.13 & $1.64[0.93-2.87]$ & 0.09 \\
\hline \multicolumn{5}{|l|}{ Facility } \\
\hline Community & reference & & reference & \\
\hline Academic & 1.12 [1.02-1.23] & 0.02 & $1.10[0.50-2.41]$ & 0.82 \\
\hline \multicolumn{5}{|l|}{ Insurance } \\
\hline Uninsured & reference & & reference & \\
\hline Insured & 1.53 [1.19-1.96] & 0.001 & $1.51[0.18-12.53]$ & 0.7 \\
\hline \multicolumn{5}{|l|}{ Income (\$) } \\
\hline$<48,000$ & reference & & reference & \\
\hline$\geq 48,000$ & 1.18 [1.08-1.30] & $<0.001$ & $1.33[0.65-2.70]$ & 0.43 \\
\hline \multicolumn{5}{|l|}{ Population } \\
\hline Urban & reference & & reference & \\
\hline Rural & $0.64[0.46-0.89]$ & 0.009 & 1.32 [0.15-12.09] & 0.8 \\
\hline \multicolumn{5}{|c|}{ Comorbidity Score } \\
\hline 0 & reference & & reference & \\
\hline$\geq 1$ & $0.95[0.85-1.06]$ & 0.32 & $1.14[0.52-2.50]$ & 0.75 \\
\hline \multicolumn{5}{|l|}{ Year } \\
\hline 2004-2012 & reference & & reference & \\
\hline 2013-2015 & 1.39 [1.27-1.53] & $<0.001$ & $0.98[0.53-1.82]$ & 0.95 \\
\hline \multicolumn{5}{|l|}{ Grade } \\
\hline $1-2$ & reference & & reference & \\
\hline 3 & $0.67[0.56-0.80]$ & $<0.001$ & $1.77[0.78-4.02]$ & 0.17 \\
\hline \multicolumn{5}{|l|}{ Clinical T stage } \\
\hline T1-T2 & reference & & reference & \\
\hline T3 & $0.65[0.54-0.78]$ & $<0.001$ & $0.40[0.14-1.13]$ & 0.08 \\
\hline T4 & $0.28[0.21-0.37]$ & $<0.001$ & $0.20[0.06-0.75]$ & 0.02 \\
\hline \multicolumn{5}{|l|}{ Clinical N stage } \\
\hline No & reference & & reference & \\
\hline N1 & $0.83[0.75-0.91]$ & $<0.001$ & $1.13[0.53-2.43]$ & 0.75 \\
\hline
\end{tabular}




\section{Cureus}

N2

Radiation Completion to Surgery (days)

$$
\leq 56
$$

$>56$

Total Dose (Gy)

$\leq 50.4$

$>50.4$

Length Radiation Treatment (days)

$$
\leq 40
$$

$>40$

Diagnosis to Chemo Initiation (days)

$\leq 33$

$>33$

0.69 [0.56-0.84]

$<0.001$

$1.23[0.50-3.04]$

0.65

reference

$1.22[1.12-1.34]$

reference

$0.94[0.82-1.07]$

0.34

eference

$0.52[0.15-1.78]$

0.3

reference

0.97 [0.88-1.06]

0.47

reference

0.99 [0.53-1.86]

0.98

reference

$1.29[1.17-1.41]$ reference

$1.26[0.72-2.20]$

0.42

\section{TABLE 3: Patient and treatment characteristics associated with $\mathrm{pCR}$ stratified by treatment group}

nCRT: neoadjuvant chemoradiation; TNT: total neoadjuvant therapy; OR: odds ratio; Cl: confidence interval; pCR: pathologic complete response; NAR: neoadjuvant rectal cancer

Median follow-up was 38 months (TNT group) versus 54 months (nCRT group). The five-year OS was significantly better for the TNT group (76.2\% vs. 69.9\%) on univariate analysis (HR=0.58, $\mathrm{p}=0.002)$, but not

\begin{tabular}{|c|c|c|}
\hline Prognostic Factor & HR [95\% Cl] & P-value \\
\hline \multicolumn{3}{|l|}{ Group } \\
\hline nCRT & reference & \\
\hline TNT & 0.58 [0.41-0.82] & 0.002 \\
\hline \multicolumn{3}{|l|}{ Age (years) } \\
\hline$\leq 65$ & reference & \\
\hline$>65$ & 1.84 [1.73-1.96] & $<0.001$ \\
\hline \multicolumn{3}{|l|}{ Gender } \\
\hline Male & reference & \\
\hline Female & $0.84[0.79-0.90]$ & $<0.001$ \\
\hline \multicolumn{3}{|l|}{ Facility } \\
\hline Community & reference & \\
\hline Academic & $0.81[0.76-0.86]$ & $<0.001$ \\
\hline \multicolumn{3}{|l|}{ Insurance } \\
\hline Uninsured & reference & \\
\hline Insured & 0.91 [0.78-1.05] & 0.19 \\
\hline \multicolumn{3}{|l|}{ Income (\$) } \\
\hline$<48,000$ & reference & \\
\hline$\geq 48,000$ & 0.84 [0.78-0.89] & $<0.001$ \\
\hline
\end{tabular}
on PMA (adjusted HR=0.79, p=0.19) (Table 4, Figure 2). 


\section{Cureus}

Population

Urban

Rural

Comorbidity Score

0

$\geq 1$

Year

2004-2012

2013-2015

Grade

$1-2$

3

Clinical T stage

T1-T2

T3

T4

Clinical N stage

NO

N1

N2

Pathologic T Stage

pTO

pT1

pT2

pT3

pT4

Pathologic T Response

pT+

pTO

Pathologic N Stage

pNO

$\mathrm{pN} 1$

pN2

Pathologic N Response

$\mathrm{pN}+$

pNO

pCR

ypT+ or ypN+

ypTONO reference

$1.04[0.85-1.26] \quad 0.72$

reference

$1.47[1.37-1.58] \quad<0.001$

reference

$0.84[0.76-0.93] \quad 0.001$

reference

$1.61[1.48-1.76] \quad<0.001$

reference

$1.29[1.09-1.52] \quad 0.002$

$2.19[1.81-2.64] \quad<0.001$

reference

$0.98[0.91-1.04] \quad 0.49$

$1.23[1.07-1.40] \quad 0.003$

reference

$1.23[1.02-1.47] \quad 0.026$

$1.35[1.19-1.55] \quad<0.001$

$2.48[2.20-2.80] \quad<0.001$

$4.63[3.95-5.43] \quad<0.001$

reference

$0.48[0.43-0.54]<0.001$

reference

$1.70[1.58-1.84] \quad<0.001$

$2.73[2.48-2.99] \quad<0.001$

reference

$0.47[0.43-0.52] \quad<0.001$

reference

$0.46[0.40-0.52] \quad<0.001$ 


\section{Cureus}

Pathologic Response

$\begin{array}{lll}\text { ypT+N+ } & \text { reference } & \\ \text { pTON+ } & 0.39[0.24-0.66] & <0.001 \\ \text { pT+NO } & 0.49[0.45-0.54] & <0.001 \\ \text { pTONO } & 0.31[0.25-0.38] & <0.001\end{array}$

NAR Score

$<8$

8-16

$>16$

Radiation Completion to Surgery (days)

$\leq 56$

$>56$

Total Dose (Gy)

$\leq 50.4$

$>50.4$

Length Radiation Treatment (days)

$\leq 40$

$>40$

Diagnosis to Chemo Initiation (days)

$\leq 33$

$>33$ reference

$\begin{array}{ll}1.53[1.38-1.70] & <0.001 \\ 2.60[2.34-2.89] & <0.001\end{array}$

reference

$1.16[1.09-1.24] \quad<0.001$

reference

$1.11[1.02-1.21] \quad 0.018$

reference

$1.23[1.15-1.31] \quad<0.001$

reference

$1.01[0.95-1.08] \quad 0.76$

TABLE 4: Univariate Cox proportional hazards model for overall survival

nCRT: neoadjuvant chemoradiation; TNT: total neoadjuvant therapy; HR: hazard ratio; Cl: confidence interval; pCR: pathologic complete response; NAR: neoadjuvant rectal cancer 


\section{Cureus}

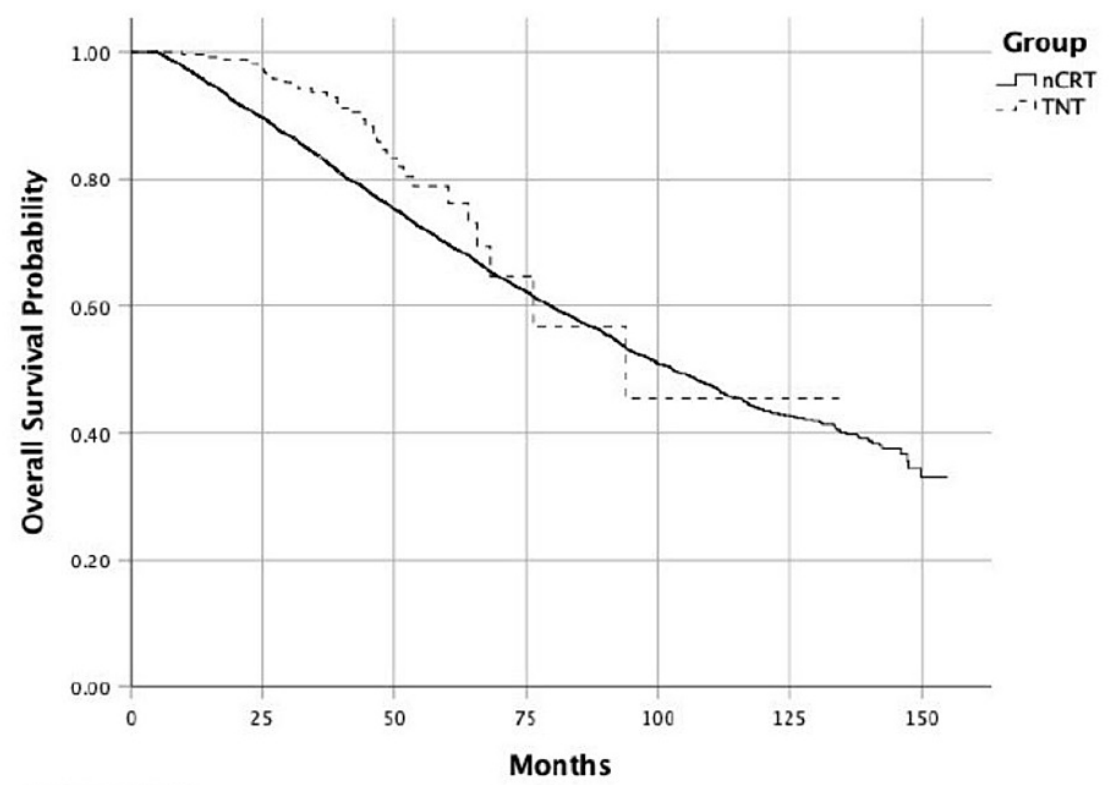

Number at Risk:

nCRT: 13530

10553

5614

2305

983

348

20

TNT: 264

220

60

7

3

1

0

FIGURE 2: Overall survival by treatment group

Older age, a co-morbidity score $\geqslant 1$, high-grade disease, and advanced $\mathrm{T}$ and $\mathrm{N}$ stage were associated with worse overall survival $(\mathrm{p}<0.001)$, whereas female gender, treatment at an academic facility, and higher income were associated with improved survival (Table 4). The five-year OS for patients with a pathologic complete response versus residual disease was $85.1 \%$ vs. $67.5 \%$, respectively ( $H R=0.46, \mathrm{p}<0.001)$. Pathologic complete nodal (ypT+N0) and primary tumor response (ypTON+) were both associated with improved survival compared to residual primary tumor and nodal disease $(\mathrm{ypT}+\mathrm{N}+)$ (Table 4). There was no difference in survival between $\mathrm{pCR}$ of the primary tumor versus nodal disease (HR=1.26, 95\%CI: 0.75-2.10, $\mathrm{p}=0.38$ ).

Figure 3 demonstrates overall survival based on pathologic response to treatment. 


\section{Cureus}

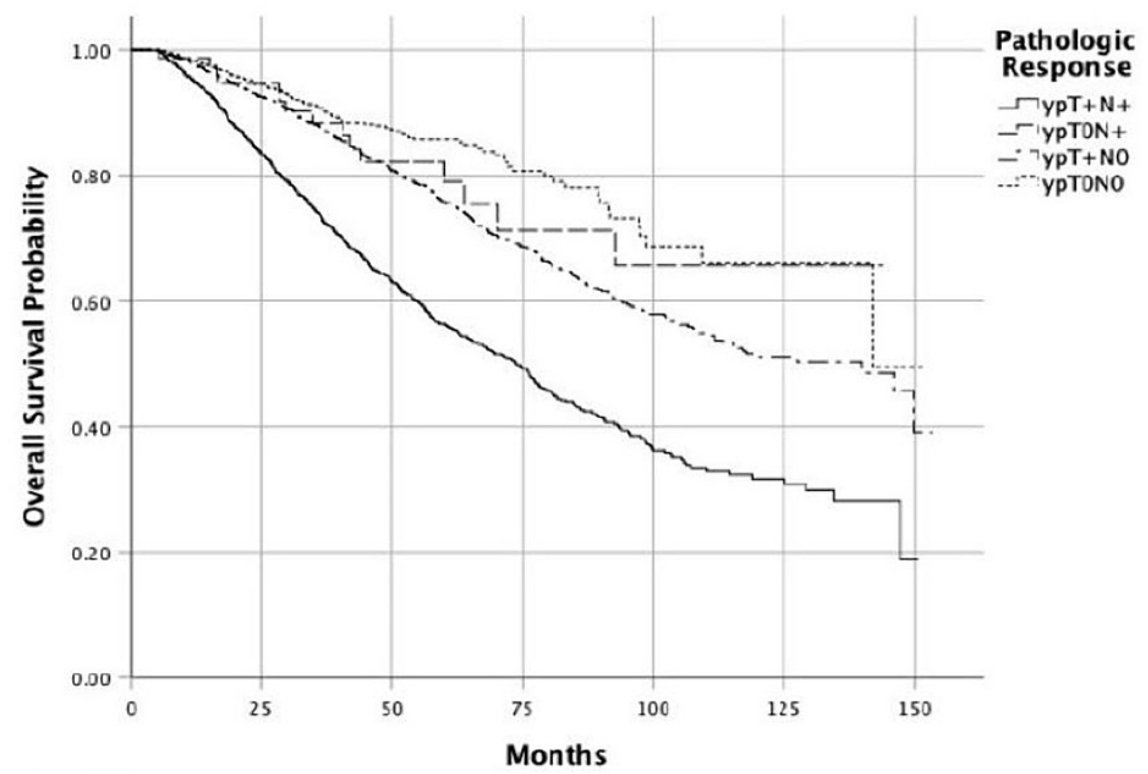

Number at Risk:

ypT+N+: 2234

ypTON+: 78

YPT+NO: 3197

1639

780

317

ypTONO: 831

66

2530

32

16

118

9

221

40

$\begin{array}{ll}38 & 1 \\ 1 & 0 \\ 81 & 5 \\ 17 & 1\end{array}$

FIGURE 3: Predicted survival based on pathologic response to therapy

When stratified by clinical stage, we found that patients with cT3 or cN1 disease (adjusted $\mathrm{HR}=0.6, \mathrm{p}=0.038$, and adjusted HR $=0.58, \mathrm{p}=0.049$, on PMA, respectively) had improved OS when treated with TNT. Patients with cT4 disease or $\mathrm{cN} 2$ disease were not found to have improved OS with TNT ( $\mathrm{p}=0.853$ vs. $\mathrm{p}=0.791$, respectively) (Table 5).

\begin{tabular}{|c|c|c|c|c|c|c|c|c|}
\hline \multirow{2}{*}{ Group } & \multicolumn{2}{|l|}{ cT3 } & \multicolumn{2}{|l|}{ cT4 } & \multicolumn{2}{|l|}{ cN1 } & \multicolumn{2}{|l|}{ cN2 } \\
\hline & $\begin{array}{l}\text { Propensity Matched } \\
\text { HR }[95 \% \mathrm{Cl}]\end{array}$ & $\begin{array}{l}\text { P- } \\
\text { value }\end{array}$ & $\begin{array}{l}\text { Propensity Matched } \\
\text { HR }[95 \% \mathrm{Cl}]\end{array}$ & $\begin{array}{l}\mathrm{P} \text { - } \\
\text { value }\end{array}$ & $\begin{array}{l}\text { Propensity Matched } \\
\text { HR }[95 \% \mathrm{Cl}]\end{array}$ & $\begin{array}{l}\mathrm{P} \text { - } \\
\text { value }\end{array}$ & $\begin{array}{l}\text { Propensity Matched } \\
\text { HR }[95 \% \mathrm{CI}]\end{array}$ & $\begin{array}{l}\mathrm{P} \text { - } \\
\text { value }\end{array}$ \\
\hline nCRT & \multicolumn{2}{|l|}{ reference } & \multicolumn{2}{|l|}{ reference } & \multicolumn{2}{|l|}{ reference } & \multicolumn{2}{|l|}{ reference } \\
\hline TNT & $0.60[0.37-0.97]$ & 0.038 & $0.94[0.51-1.74]$ & 0.853 & 0.58 [0.34-1.00] & 0.049 & $0.91[0.46-1.82]$ & 0.791 \\
\hline
\end{tabular}

\section{Discussion}

We used the NCDB to evaluate a large patient population with locally advanced rectal cancer to investigate pCR rates and OS outcomes for patients treated with TNT or nCRT. As demonstrated in previous studies, we found that TNT was more commonly utilized for patients with advanced-stage cT4 disease (17\% vs $8 \%$ ) and node-positive disease ( $20 \%$ vs $7 \%$ for N2 disease). When evaluating treatment response, we found a trend towards improved pCR with the use of TNT (17.5\% vs $14.2 \%, \mathrm{p}=0.053)$, though this was not statistically significant. No statistically significant difference was observed in five-year OS between treatment groups (TNT 76.2\% vs. nCRT 69.9\%), despite higher utilization of TNT for more advanced-stage disease. This is consistent with other NCDB analyses demonstrating no difference in overall survival between treatment groups $[13,14]$. However, when stratified by clinical stage, we found that patients with cT3 or cN1 disease had better OS when treated with TNT versus nCRT. Patients with cT4 disease or cN2 disease were not found to have improved OS with TNT ( $\mathrm{p}=0.853$ vs. $\mathrm{p}=0.791$, respectively). 
rate in patients with cT4 disease in both treatment groups. In contrast, we found that increasing nodal burden was associated with a lower pCR rate for the nCRT group whereas it was independent of pCR rate in the TNT group. When evaluating nodal $\mathrm{pCR}$, we found that the rate of pathologic complete nodal response was significantly improved with the use of TNT compared to nCRT (72\% vs 64\%). Several studies have demonstrated that pathologic tumor response rates are important prognostic factors for survival in rectal cancer [6-8,15-16], and are achieved in roughly $14-40 \%$ of patients treated with neoadjuvant therapy $[17,18]$. An exploratory analysis of the CAO/ARO/AIO-94 trial showed that both pathologic nodal response and primary tumor response after nCRT were the most important independent prognostic factors for diseasefree, metastases-free, and local relapse-free survival [8]. In concordance with these results, we found that overall survival was better for patients who achieved a pathologic complete response (five-year OS of $85 \%$ vs. $67 \%)$.

The novel finding in our study was that TNT improves the rate of nodal pCR compared to nCRT and could account for the improved overall survival observed with TNT in the subset of patients with cT3 and cN1 disease. These findings suggest that preoperative TNT for locally advanced rectal cancer may provide the most benefit for patients with less advanced-stage disease. While there are existing clinical trials comparing these treatment modalities, long-term follow-up is needed to confirm a survival benefit in this subset of patients.

There are several inherent limitations associated with analyzing retrospective data from a large cancer registry, including uncertainties in the accuracy of collected data and the potential for selection bias. Further, data collected from the NCDB database does not include pertinent prognostic information, including tumor location and tumor distance from the mesorectal fascia, which recent studies have suggested may obviate the need for neoadjuvant therapy altogether for select patients [19].

\section{Conclusions}

In our study, TNT was associated with higher nodal pCR rates, which is independently associated with improved overall survival. When compared to nCRT, TNT was associated with improved overall survival for patients with cT3 and cN1 disease. Our results suggest that while TNT is the treatment of choice for patients with more advanced disease, consideration should be made in utilizing this modality for patients with less advanced stage III rectal cancer.

\section{Additional Information \\ Disclosures}

Human subjects: Consent was obtained or waived by all participants in this study. Animal subjects: All authors have confirmed that this study did not involve animal subjects or tissue. Conflicts of interest: In compliance with the ICMJE uniform disclosure form, all authors declare the following: Payment/services info: All authors have declared that no financial support was received from any organization for the submitted work. Financial relationships: All authors have declared that they have no financial relationships at present or within the previous three years with any organizations that might have an interest in the submitted work. Other relationships: All authors have declared that there are no other relationships or activities that could appear to have influenced the submitted work.

\section{References}

1. Siegel RL, Miller KD, Jemal A: Cancer statistics, 2020. CA Cancer J Clin. 2020, 70:7-30. 10.3322/caac.21590

2. National Comprehensive Cancer Network. Rectal cancer (Version 3.2019) . (32019). Accessed: May 6, 2020: https://www.nccn.org/professionals/physician_gls/pdf/rectal.pdf.

3. Sauer R, Liersch T, Merkel S, et al.: Preoperative versus postoperative chemoradiotherapy for locally advanced rectal cancer: results of the German CAO/ARO/AIO-94 randomized phase III trial after a median follow-up of 11 years. J Clin Oncol. 2012, 30:1926-33. 10.1200/JCO.2011.40.1836

4. Taal BG, Van Tinteren H, Zoetmulder FA: Adjuvant 5FU plus levamisole in colonic or rectal cancer: improved survival in stage II and III. Br J Cancer. 2001, 85:1437-43. 10.1054/bjoc.2001.2117

5. Bosset JF, Collette L, Calais G, et al.: Chemotherapy with preoperative radiotherapy in rectal cancer . N Engl J Med. 2006, 355:1114-23. 10.1056/NEJMoa060829

6. Maas M, Nelemans PJ, Valentini V, et al.: Long-term outcome in patients with a pathological complete response after chemoradiation for rectal cancer: a pooled analysis of individual patient data. Lancet Oncol. 2010, 11:835-44. 10.1016/S1470-2045(10)70172-8

7. Fokas E, Liersch T, Fietkau R, et al.: Tumor regression grading after preoperative chemoradiotherapy for locally advanced rectal carcinoma revisited: updated results of the CAO/ARO/AIO-94 trial. J Clin Oncol. 2014, 32:1554-62. 10.1200/JCO.2013.54.3769

8. Rödel C, Martus P, Papadoupolos T, et al.: Prognostic significance of tumor regression after preoperative chemoradiotherapy for rectal cancer. J Clin Oncol. 2005, 23:8688-96. 10.1200/JCO.2005.02.1329

9. Manthravadi S, Sun W, Saeed A, et al.: Total neoadjuvant therapy compared with standard therapy in locally advanced rectal cancer: A systematic review and meta-analysis. J Clin Oncol. 2019, 37:709-709. 10.1200/JCO.2019.37.4_suppl.709

10. Cercek A, Roxburgh CS, Strombom P, et al.: Adoption of Total Neoadjuvant Therapy for Locally Advanced Rectal Cancer. JAMA Oncol. 2018, 4:e180071. 10.1001/jamaoncol.2018.0071 


\section{Cureus}

11. Yothers G, George T, Petrelli NJ, et al.: Neoadjuvant rectal cancer (RC) score to predict survival: Potential surrogate endpoint for early phase trials. J Clin Oncol. 2014, 32:3533. 10.1200/jco.2014.32.15_suppl.3533

12. You N, George T, Chiang YJ, et al.: Validation of neoadjuvant rectal cancer (NAR) score as a surrogate endpoint for overall survival in real-life practice settings. J Clin Oncol. 2018, 36:3517.

10.1200/JCO.2018.36.15_suppl.3517

13. Zhu S, Brodin NP, English K, et al.: Comparing outcomes following total neoadjuvant therapy and following neoadjuvant chemoradiation therapy in patients with locally advanced rectal cancer. EClinicalMedicine. 2019, 16:23-9. 10.1016/j.eclinm.2019.09.009

14. Babar L, Bakalov V, Abel S, et al.: Temporal trends and disease characteristics associated with total neoadjuvant therapy (TNT) usage. J Clin Oncol. 2019, 37:642. 10.1200/JCO.2019.37.4_suppl.642

15. Lorimer PD, Motz BM, Kirks RC, et al.: Pathologic complete response rates after neoadjuvant treatment in rectal cancer: an analysis of the National Cancer Database. Ann Surg Oncol. 2017, 24:2095-103.

10.1245/s10434-017-5873-8

16. Dinaux AM, Amri R, Bordeianou LG, et al.: The impact of pathologic complete response in patients with neoadjuvantly treated locally advanced rectal cancer- a large single-center experience. J Gastrointest Surg. 2017, 21:1153-8. 10.1007/s11605-017-3408-z

17. Petrelli F, Trevisan F, Cabiddu M, et al.: Total neoadjuvant therapy in rectal cancer: a systematic review and meta-analysis of treatment outcomes. Ann Surg. 2020, 271:440-8. 10.1097/SLA.0000000000003471

18. Lee SH: How to achieve a higher pathologic complete response in patients with locally advanced rectal cancer who receive preoperative chemoradiation therapy. Ann Coloproctol. 2019, 35:3-8. 10.3393/ac.2019.02.17

19. Kennedy ED, Simunovic M, Jhaveri K, et al.: Safety and feasibility of using magnetic resonance imaging criteria to identify patients with "good prognosis" rectal cancer eligible for primary surgery: The phase 2 nonrandomized QuickSilver clinical trial. JAMA Oncol. 2019, 5:961-6. 10.1001/jamaoncol.2019.0186 